\title{
Assemblage métallurgique des pièces moteurs. Soudage par friction inertielle de disques de compresseur HP en superalliage de nickel
}

\author{
J.-P. FERTE
}

SNECMA, BP. 81, 91003 Evry cedex, France

\begin{abstract}
The main part of this paper describes upside metallurgical and mechanical work done at SNECMA, on inertia wetding of powder metallurgy nickel base superalloys ASTROLOY and N18, allowing appliance of this process to engine parts : Inertia welding of superalloys bads to deap microstructural changes in the H.A.Z which have been , as well as upset, correlated to process parameters, weld geometry and base material microstructure; a full mechanical testing of welds shown properties equivalent to base material ones up to $650^{\circ} \mathrm{C}$ except for fatigue crack growth behavior under specific conditions $\left(T>600^{\circ} \mathrm{C}\right.$-hold time at maximum load) which is drastically reduced for in weld plane propagation.A significant improvement of this later property has been done through post-welding heat treatment and optimization of welding parameters.

Last part of this paper summarize the main teachings gained, on the complete welding procedure, from weiding of scale one parts.
\end{abstract}

L'assemblage des disques de compresseur HP de turboréacteurs, réalisés en alliages de titane pour les parties les moins chaudes et en superalliages de nickel pour les plus chaudes, nécessite des procédés parfaitement maitrisés et devant conduire à des liaisons satisfaisant à des spécifications géométriques et mécaniques sévères.

Les procédés d'assemblage retenus par la majorité des motoristes sont : le soudage par faisceau d'électrons (F.E) et/ou le soudage par friction inertielle (F.I).

Le dimensionnement des nouveaux moteurs SNECMA a conduit cette dernière à développer et à industrialiser, en copération, un nouveau superalliage à base nickel pour disques chauds, réalisé suivant une route "métallurgie des poudres", ralliage N18 [1], présentant par rapport à l'ASTROLOY, préalablement utilisé, des gains significatifs en température d'utilisation et en résistance à la propagation des fissures. Des études internes ont montré que ces derniers alliages n'étaient pas soudables par FE pour des épaisseurs supérieures à $5 \mathrm{~mm}$, ce procédé conduisant, dans ces conditions, à des fissurations de zone fondue bcalisées dans la région d'évanouissement du faisceau. SNECMA s'est orientée, pour lassemblage des disques chauds des compresseurs HP, vers le procédé Fl, sur la base : d'une part expérience industrielle très satisfaisante, dans le soudage d'arbres de turbine en MARAGING 250, ayant démontré la reproductibilité et la simplicité du programme d'assurance qualité associé à ce procédé, et d'autre part de rabsence de zone fondue et de ses défauts potentiels associés.

Cet article résume les différents travaux préalables réalisés, tout d'abord sur ASTROLOY puis sur N18, à SNECMA avant le lancement de la phase industrielle. Ces derniers, essentiellement expérimentaux, ont été supportés par des actions de modélisation du procédé en collaboration avec le CEMEF [2] et le centre PM FOURT de HEcole des Mines de Paris [3].

\section{I - RAPPELS SUR LE PROCEDE - CRITERES}

Une schématisation du procédé est présentée à la figure 1. Les paramètres opératoires du soudage sont : le moment d'inertie de l'ensemble tournant, la vitesse de rotation initiale de ce dernier et la force de pression appliquée. Ces derniers sont généralement remplacés, au stade des études, par des variables à caractère plus général : rénergie cinétique initiale par unité de surface (énergie surfacique), la vitesse linéaire initiale de soudage, la pression appliquée. Une grandeur essentielle, résultante de ropération, est la réduction de longueur des pièces après assemblage ou consommation-matière. 


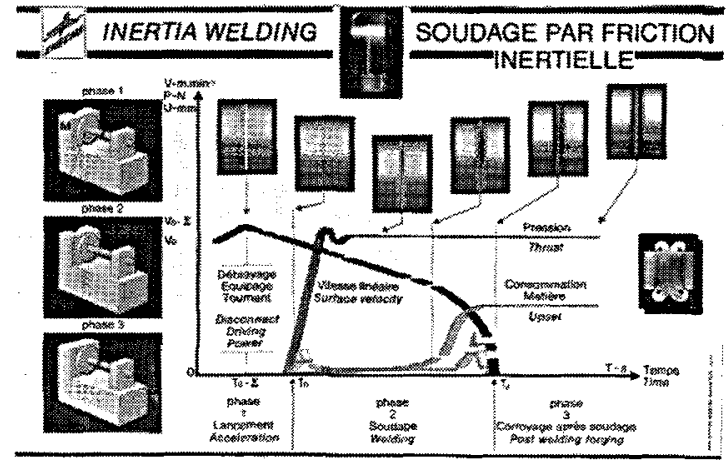

figure 1: schéma de principe du soudage F.J

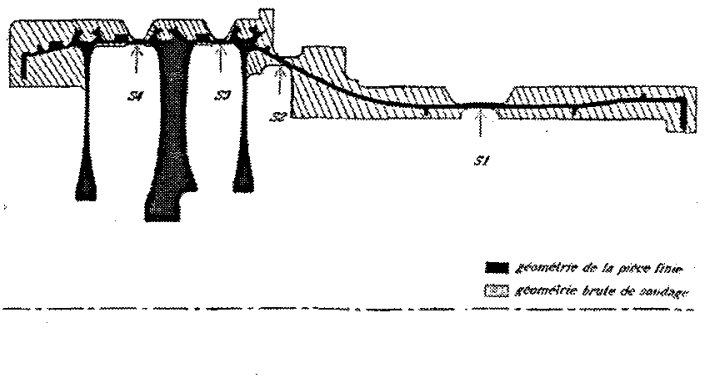

figure 2: schéma d'un rotor soudé F.I

Le schéma d'un rotor de compresseur HP ainsi que sa géométrie type à létat brut de soudage sont illustrés à la figure 2. On y observe une des caractéristiques contraignantes de ce procédé : la nécessité de consenver avant soudage des surépaisseurs importantes au droit des prises de pièces pour pouvoir supporter les efforts élevés de serrage et de pression.

Les critères essentiels à satisfaire sont de plusieurs ordres :

- géométriques : la consommation-matière, la coaxialité et le défaut de perpendicularité sont tolérancés à $\pm 0.25 \mathrm{~mm}$, pour un diamètre moyen de soudage de $400 \mathrm{~mm}$,

- métallurgiques : absence de défauts critiques de type fissure ou alignement d'oxyde,

- mécaniques : dans le cas des rotors de compresseur HP actuels, les températures peuvent atteindre $700^{\circ} \mathrm{C}$ et les propriétés dimensionnantes sont la tenue à la fatigue oligocyclique et la résistance à la propagation des fissures.

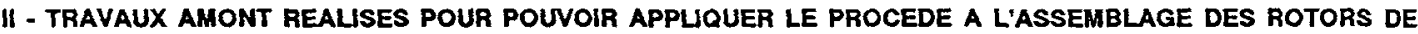 COMPRESSEUR HP EN ALLAGE N18}

Ces travaux ont pour objectifs principaux de déterminer les plages de paramètres opératoires de soudage, les trailements thermiques et opérations d'usinage post-soudage permettant de satisfaire les critères précédemment définis.

II - 1 Influence de la géométrie initiale des pièces et de la microstructure de départ sur la consommation-matière et la microstructure des liaisons

Les travaux ont été réalisés sur ASTROLOY et N18 transformés et traités thermiquement suivant des conditions standards (filage des poudres, forgeage isotherme) et conduisant à une structure équiaxe fine (5-10 $\mu \mathrm{m})$ et à une double précipitation de phase durcissante $\gamma$ '. Les essais de soudage ont été réalisés, principalement à partir d'éprouvettes tubulaires de diamètre moyen $90 \mathrm{~mm}$, pour une plage d'épaisseur à souder allant de 6 à $13 \mathrm{~mm}$, sur une machine M.T.l - M300.

La figure 3 décrit les principales caractéristiques macrographiques et micrographiques d'une liaison N18/N18 d'épaisseur 7.5 $\mathrm{mm}$ réalisée dans des conditions représentatives et observée à létat brut de soudage. La microstructure évolue fortement entre le plan de joint et le métal de base sur une distance d'environ $0.5 \mathrm{~mm}$. On observe tout d'abord, en partant du plan de joint, une zone centrale apparaissant blanche en micrographie optique, confinée dans lépaisseur de la pièce et de largeur totale $0.1-0.15 \mathrm{~mm}$, où la phase $\gamma_{\text {; }}$, présente sous forme de précipités primaires $(2-3 \mu \mathrm{m})$ et secondaires $(0.3 \mu \mathrm{m})$ dans le métal de base, a complétement été remise en solution. Une observation plus fine de cette zone blanche en M.E.T met en évidence une population tertiaire de précipités $\mathscr{\gamma}$ ' ultrafins (100 $\AA$ ) [4] produits lors du refroidissement naturel très rapide propre à ce procédé; celle-ci est bordée de deux zones apparaissant grises qui se probngent dans les bourrelets. Dans ces dernières, les précipités durcissants primaires restent présents alors que les précipités secondaires ont été remis en solution. On observe ensuite, avant de retrouver le métal de base, une dernière zone affectée correspondant à la dissolution décroissante des précipités secondaires.

Une observation à fort grossissement permet d'identifier des zones présentant des défauts de cavitation correspondant à des décohésions, aux interfaces $\not / \gamma^{\prime}$, de taille de rordre du $\mu \mathrm{m}$ : ces zones sont localisées en périphérie, près des bourrelets, à linterface zone grise/zone affectée, et les défauts correspondant sont présents sur une profondeur de 1 - $1.5 \mathrm{~mm}$ à partir de la surface. 


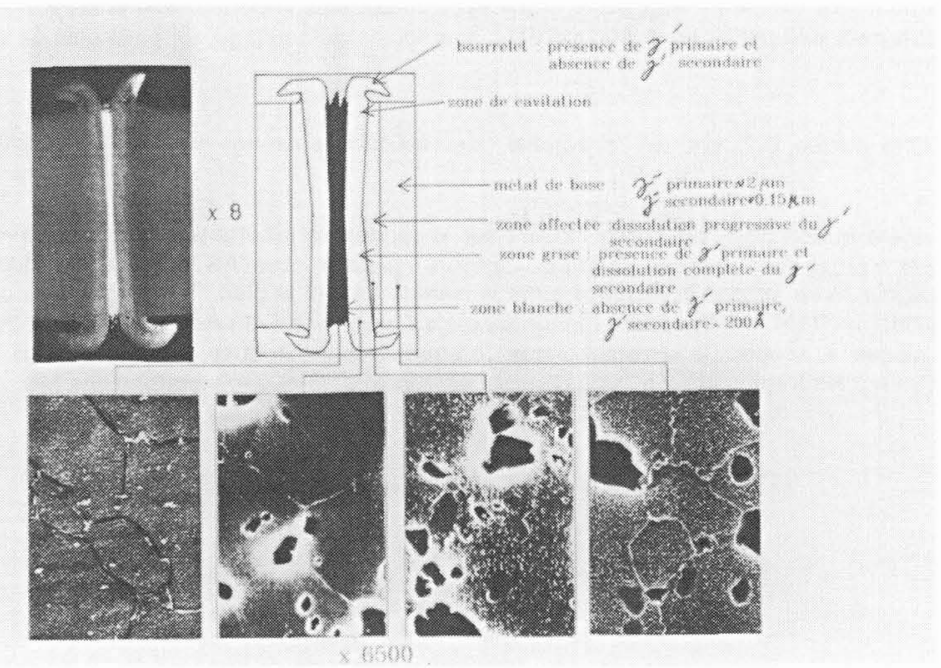

figure 3 : macro et microstructure d'une liaison N18/N18.

Des essais de cyclage thermique rapide sur ASTROLOY [3] ont permis de déterminer les températures de solvus $\gamma^{\prime}$ pour les précipités primaires et secondaires, dans des conditions représentatives des conditions de soudage. Ces derniers résultats, associés à des mesures pyrométriques de température de surface et à des mesures conventionnelles par thermocouples, ont permis de tracer (figure 4) révolution de la température maximale vue par ralliage en fonction de la distance finale au plan de joint. On observe d'une part, que la température d'interface correspond sensiblement à celle du solidus du N18 et d'autre part, sur les premiers $1 / 10 \mathrm{~mm}$, un grandient de rordre de $800^{\circ} \mathrm{C} / \mathrm{mm}$ devant générer des contraintes résiduelles importantes du fait de reffet de trempe produit par le refroidissement très rapide observé après soudage ; la vitesse de $\infty$ dernier est estimée, à partir de la taille du $\gamma^{\prime}$ tertiaire, à environ $8000^{\circ} \mathrm{C} / \mathrm{min}$ [5].
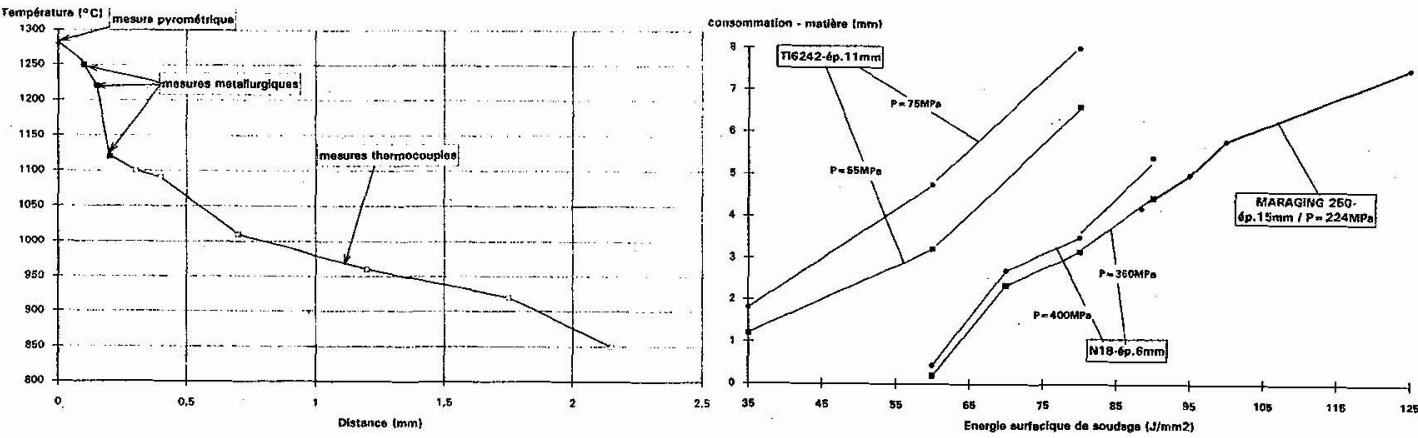

figure 4 : liaison ASTROLOY/ASTROLOY, évolution de la température maximale le long de Paxe de la soudure

figure 5 : liaison $N 18 / N 18$, influence de l'énergie surfacique de soudage sur la consommation-matière résultante

L'influence des paramétres de soudage sur la consommation-matière, pour une épaisseur soudée de $6 \mathrm{~mm}$, est illustrée à la figure 5. On met en évidence, le niveau de pression élevé nécessaire au soudage des superalliages de nickel, comparativement aux alliages de titane et aux aciers et l'existence d'un seuil minimal d'énergie nécessaire à lapparaition de la consommation-matière et correspondant à lénergie thermique nécossaire pour porter la proximité du plan de soudage à plus de $1100^{\circ} \mathrm{C}$. La figure 6 illustre linfluence du diamètre moyen et de lépaisseur à souder sur la relation C.M/énergie surfacique de soudage. On remarque que, à même énergie surfacique, la C.M augmente quand répaisseur diminue ou brsque le diamètre moyen augmente; $c$ type d'influence est mis en évidence de la même manière sur les alliages de titane et retrouvé par la modélisation numérique [2] ; il provient, pour une part, de la raideur plus importante du bourrelet intérieur 
(comparativement au bourrelet extérieur) dont Peffet relatif est d'autant plus important que le rapport épaisseur/diamètre est élevé et, pour une autre part, des pertes énergétiques dans la machine de soudage, en particulier au niveau des roulements de broche.

La dispersion de la C.M est de $0.2 \mathrm{~mm}$ sur rensemble des résultats, pour des conditions de soudage représentatives données.

Un paramètre difficilement quantifiable, mais très infiuent sur le rendement énergétique de Yopération de soudage, est la géométrie de la soudure à proximité du plan de soudage. La figure 7 illsutre, pour PASTROLOY, la réduction drastique du rendement énergétique observée lorsque la distance entre le plan de joint et le plan de serrage de la pièce (A : porte à faux) augmente. Ces résultats mettent en évidence limportance des pertes potentielles d'énergie, consommée sous forme vibratoire, dans les pièces à souder. Il en résulte que l'on recherche à réduire au minimum le porte à faux et plus généralement, en jouant sur les formes et/ou à laide d'outillages annexes, le niveau vibratoire des pièces à souder.
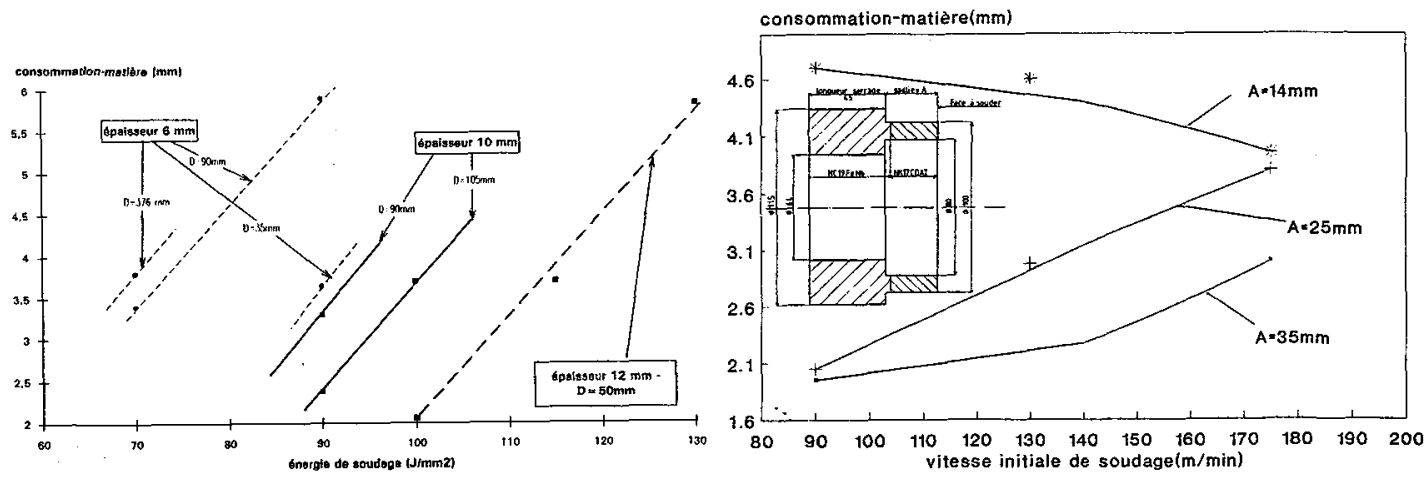

figure 6 : liaison N1B/N18, influence de rénergie, de l'épaisseur à souder et du diamètre moyen sur la fau consommation-matìre

figure 7 : liaison ASTROLOY/ASTROLOY, influence du porte à faux et de la vitesse initiale de soudage sur la C.M résultante (ép. $10 \mathrm{~mm}$ - par, de soudage standards)

La dimension des précipités durcissants secondaires dans le métal de base (N18 ou ASTROLOY) est fonction principalement de la vitesse de refroidissement obtenue brs de la trempe suivant le traitement thermique de mise en solution : elle peut varier, suivant le milieu de refroidissement et la géométrie de la pièce, d'environ 0.1 à 0.4 um. La figure 8 illustre, pour MASTROLOY, Pinfluence de la taille de ces précipités sur la C.M obtenue après soudage. L'augmentation de cette dernière s'explique par la réduction de la température de remise en solution brsque la taille diminue [3], ce qui conduit, pour un gradient de température supposé constant à proximité du plan de joint, à une augmentation de la dimension de la zone grise dans laquelle se bcalise la déformation.

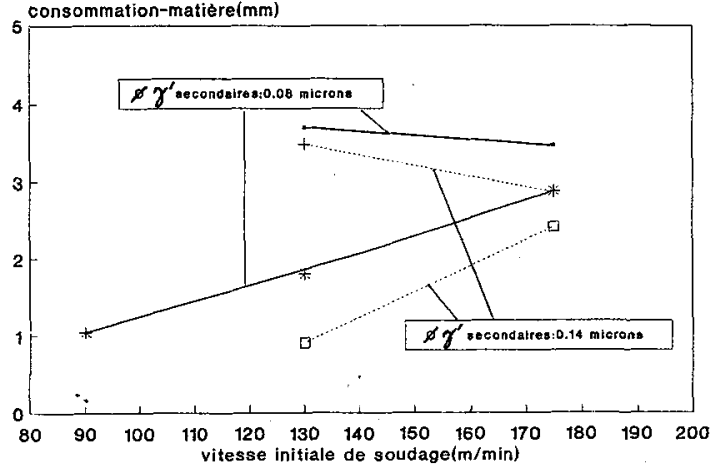

figure 8 : liaison ASTROLOY/ASTROLOY, influence de la dimension des précipités durcissants secondaires sur la consommation-matière résultante.

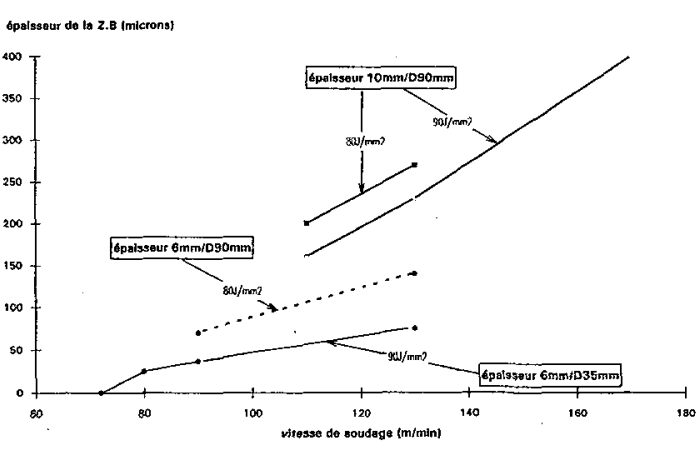

figure 9 : liaison $N 18 / N 18$, influence des paramètres opératoires sur l'épaisseur de la zône blanche centrale. 
Les dimensions des zones affectées (blanche, grise) varient aussi significativement avec la géométrie des liaisons et les paramètres de soudage. L'effet principal de ces derniers est observé sur lépaisseur de la zone blanche centrale (figure 9) : la réduction de la vitesse intiale de soudage, à énergie surfacique constante, conduit à une diminution systématique de cette zone, d'autant plus que lépaisseur à souder est faible; en deça d'une certaine vitesse critique, cette zone blanche peut disparaitre.

La profondeur de la zone de cavitation reste sensiblement constante, 1 à $1.5 \mathrm{~mm}$, sur lénsemble de la plage opératoire évalúée. De même rentaille entre bourrelets, qui pénètre à lintérieur de la pièce au niveau de la zone blanche, reste sensiblement constante et égalè à $0.7 \mathrm{~mm}$.

Une consommation-matière minimale est en général considérée comme nécessaire pour expulser dans le bourrelet la matière correspondant à la surface de soudage initiale. Nous avons mis en évidence, dans le cas du N18, pour des C.M < $1 \mathrm{~mm}$, des plages d'oxyde d'hafnium significatives. présentes au niveau du plan de joint, à proximité des bourrelets, et provenant de Yoxydation du N18 dans les premiers instants du soudage oủ les surfaces à souder ne sont pas entièrement en contact. Ces défauts ne sont plus observés pour des C.M > $2 \mathrm{~mm}$. Ce type de défaut est à rapprocher des contaminations de surface observées à faible C.M lors du soudage FI du TA6V [6]

\section{II-2 Propriétés mécaniques des liaisons N18/N18 - traitements thermiques post-soudage}

L'essentiel des caractérisations mécaniques des liaisons a été fait après le traitement thermique past-soudage correspondant au revenu standard du $N 18: 700^{\circ} \mathrm{C}-24 \mathrm{~h}+800^{\circ} \mathrm{C}-4 \mathrm{~h}$. L'évolution de la microdureté à travers une liaison type, voir figure

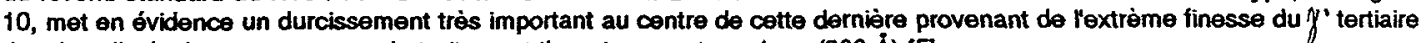
dont la taille évolue peu au cours du traitement thermique post-soudage (300 A) [5].

La caractérisation mécanique des liaisons d’épaisseurs 6 et $10 \mathrm{~mm}$, réalisées suivant diftérentes conditions de soudage représentatives de pièces réelles, à $650^{\circ} \mathrm{C}$, en traction, fluage-rupture, fatigue oligocyclique (cycle sinus, $R$ sigma = 0 ) montre que les ruptures sont le plus généralement bcalisées dans le métal de base et les résultats sont de même niveau que ceux mesurés sur ce dernier. Une analyse plus fine montre cependant que les soudures effectuées aux vitesses les plus élevées étudiées, et donc ayant les dimensions de zone affectée les plus fortes, rompent, en traction et en fluage, au niveau de la liaison, les caractéristiques mécaniques restant du même niveau que celles du métal de base, à l'exception de la ductilité.

La caractérisation de la tenue à la propagation de fissures courtes dans le plan de soudage des liaisons $N 18 / N 18$ a mis en évidence un abattement très important, par rapport au métal de base, de cette propriété lorsque la température dépasse $600^{\circ} \mathrm{C}$ et que le cycle de chargement est trapezoidal, incluant un temps de maintien de 90 à 300 s à la charge maximaie. Ces résultats, présentés à la figure 11, sont constants pour toutes les conditions de soudage étudiées et sont vraisemblablement reliés à la finesse de la précipitation $\gamma^{\prime}$ 'et à Pabsence de précipités intergranulaires dans la zone blanche centrale après le traitement thermique de revenu standard.

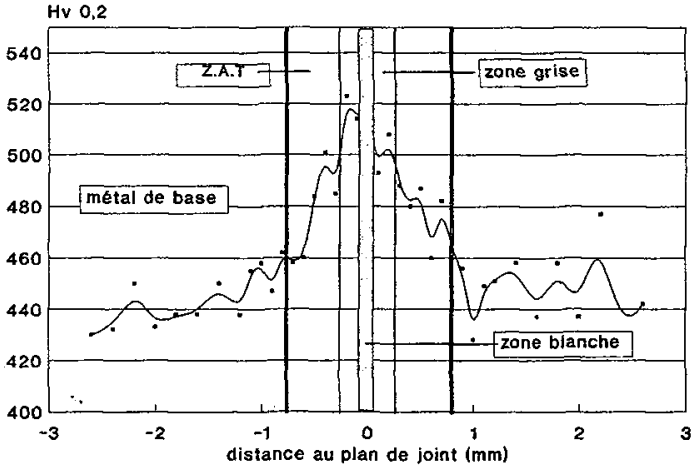

figure 10 : liaison N18/N18, évolution de la dureté à travers la liaison (ép. 6mm-par. soudage stds.- revenu postsoudage : $\left.700^{\circ} \mathrm{C}-24 \mathrm{H}+800^{\circ} \mathrm{C}-4 \mathrm{H}\right)$.

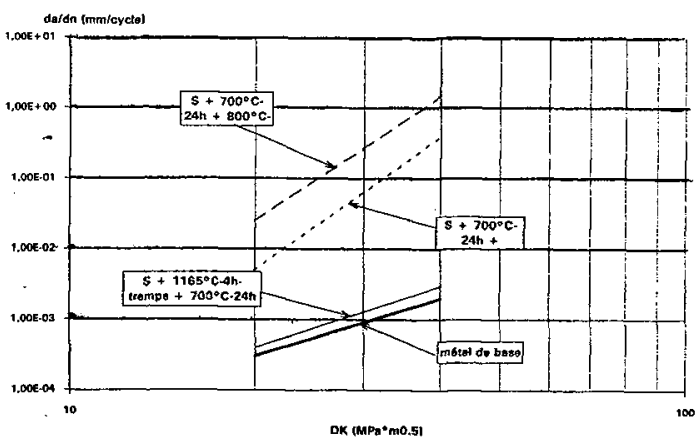

figure 11 : liaison $N 18 / N 18$, infiuence du revenu postsoudage sur la vitesse de propagation des fissures dans la zône blanche $\left(650^{\circ} \mathrm{C}\right.$ - cycle trapèze:1.5-90-1.5s).

Une amélioration sensible a été apportée (figure 11), en augmentant la durée du revenu post-soudage à $800^{\circ} \mathrm{C}$ de $4 \mathrm{~h}$ à $8 \mathrm{~h}$, qui est attribuée à leffet bénéfique de la précipitation induite de carbure de $\mathrm{Cr}$-Mo dans les joints de grains (figure 12). Un retraitement complet de la liaison après soudage, comprenant la mise en solution et les revenus, permel d'obtenir dans be centre de la liaison une structure de précipités bimodale dont les tailles sont voisines de celles du métal de base (figure 12) et de régénérer la tenue en propagation des fissures dans le plan de joint (figure 11). Ce traitement thermique complet n'est 
cependant pas envisageable car il conduit à un important grossissement des grains en bordure de ZAT, du fait de l'écrouissage résiduel présent à cet endroit après soudage; par ailleurs, son application sur pièce conduirait à des distorsions trop importantes des parties finies d'usinage. L'orientation potentielle à envisager est un traitement thermique local des soudures à une température inférieure à celle de recristallisation.
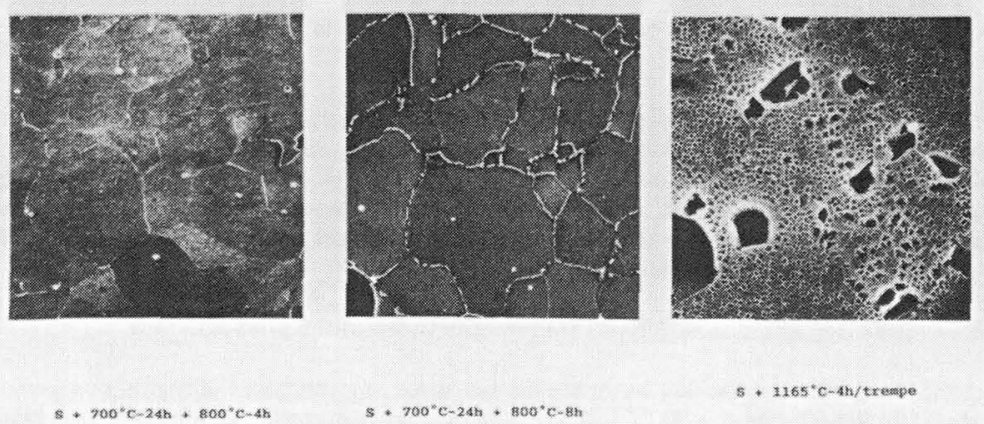

$8+700^{\circ} \mathrm{C}-24 \mathrm{ta}+800^{\circ} \mathrm{C}-4 \mathrm{n}$

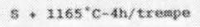

- $700^{\circ} \mathrm{C}-24 \mathrm{~h}+800^{\circ} \mathrm{c}-4 \mathrm{~h}$

figure 12 : liaison $\mathrm{N} 18 / \mathrm{N} 18$, influence du traitement thermique post-soudage sur la microstructure de la zône blanche $(\times 6500)$.

Le mode de fissuration dans le plan de soudage correspond, pour les soudures interdisques, à une sollicitation suivant laxe du moteur qui n'est généralement pas la solicitation principale appliquée à la liaison. Cette dernière est principalement soumise à une contrainte tangentielle qui conduit à une propagation de fissure potentielle perpendiculairement au plan de joint. On observe dans $c \theta$ cas, pour une dimension de zone blanche de $125 \mu \mathrm{m}$ et pour une sollicitation de type trapézoïdale, une durée de vie nettement plus importante que brs d'une propagation dans le plan de soudage (figure 13). La fissure se propage, de facon anisotrope, tout d'abord dans la zone blanche à faibles caractéristiques, puis dans le métal de base à caractéristiques élevées. II paraît ainsi que la réduction de répaisseur de la zone centrale, par un choix judicieux des paramètres de soudage, voir figure 9 , est un objectif à atteindre.

Le niveau des contraintes résiduelles, tangentielle et axiale, présentes dans une soudure F.I - N18/N18 est illustré à la figure 14 [8]. Les mesures, faites à partir de la surface externe de rassemblage suivant une méthode R.X, montrent, associées à desrelevés géométriques, que la contrainte tangentielle est de compression et que la contrainte axiale est de compression en surface externe mais de traction en surface interne; cette distribution de la contrainte axiale correspond à une flexion due au rétreint thermique imposé par la zone centrale lors de son refroidissement de $1280^{\circ} \mathrm{C}$ jusqu'à la température ambiante (figure 4). La figure 14 met en évidence Peffet de relaxation bénéfique, en particulier au niveau de la contrainte tangentielle, du traitement thermique post-soudage incluant le revenu de $8 \mathrm{~h}$ à $800^{\circ} \mathrm{C}$.
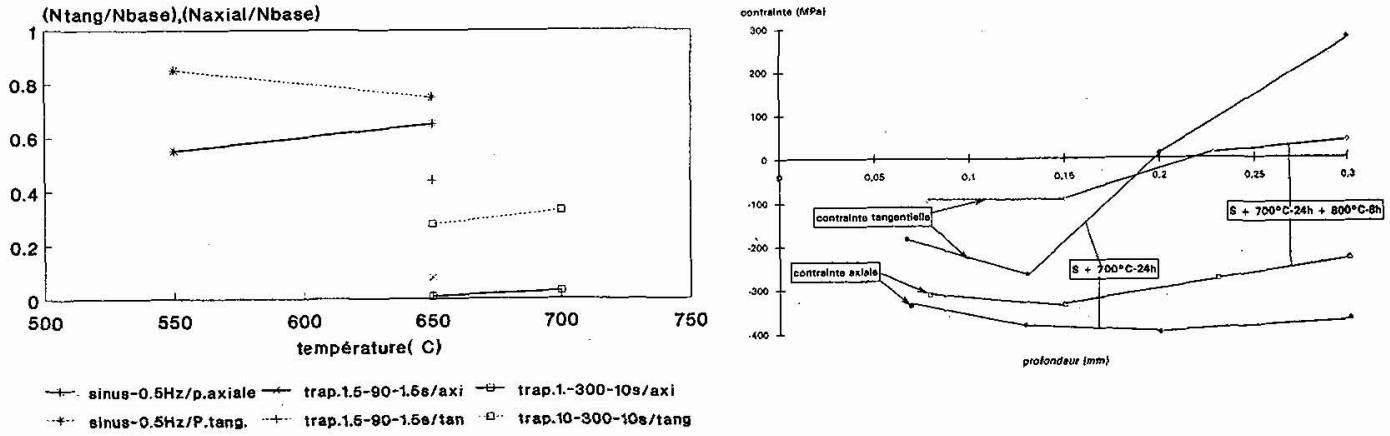

figure 13: liaison N18/N18, influence de la température, du type de sollicitation mécanique (axiale ou tangentielle) ot du mode de chargement (sinus ou trapèze) sur la durée de vie en propagation de fissure. figure 14 : liaison $N 18 / N 18$, contraintes résiduelles dans les liaisons, influence du traitement thermique past-soudage. 
La machine utilisée par SNECMA pour le soudage des rotors, M480 de fabrication M.T.I, est présentée à la figure 15 ; ses principales caractéristiques sont les suivantes : énergie maximale disponible $2700 \mathrm{~kJ}$, force de poussée maximale $4070 \mathrm{kN}$, diamètre maximal de prise de pièce $-600 \mathrm{~mm}$.

Les résultats des études à échelle réduite ont été appliqués à la réalisation de pièces de mise au point échèlle 1 en N18, ce qui a permis de préciser et de valider notre connaissance des différents éléments de la gamme de fabrication d'un rotor de compresseur HP. Les principaux résultats de cette mise au point sont présentés ci-après.

\section{Outillages et géométrie des pièces à souder}

L'assemblage d'un rotor se fait par soudages successifs des différentes pièces constitutives (S1 --> S4 - figure 2). L'état géométrique des pièces, avant soudage, est le plus proche possible des cotes finies en tenant compte de la nécessité d'assurer une grande précision de positionnement de ces dernières qui doivent par ailleurs supporter des efforts de pression axiale et de serrage importants, respectivement de lordre de 4000 et $15000 \mathrm{kN}$; ces raisons conduisent à laisser de fortes surépaisseurs au droit des surfaces de serrage et des faces d'appui. Ces mêmes raisons peuvent aussi conduire à utiliser des outillages annexes, placés à lintérieur des pièces à souder, pour contrebalancer ces efforts de serrage.

La géométrie proche du plan de joint est définie en cherchant à réduire le porte à faux (figure 7 ), pour minimiser les pertes énergétiques de forme vibratoire, tout en laissant une place suffisante à la réalisation des contrôles non destructífs. L'épaisseur à souder sera sensiblement plus importante que celle de la pièce finie car elle doit intégrer la surépaisseur correspondant aux défauts de cavitation (figure 3) et celle correspondant aux zones mortes propres au contrôle ultrasonore ; ceci conduit à rajouter typiquement $4 \mathrm{~mm}$ à lépaisseur de la pièce finie.

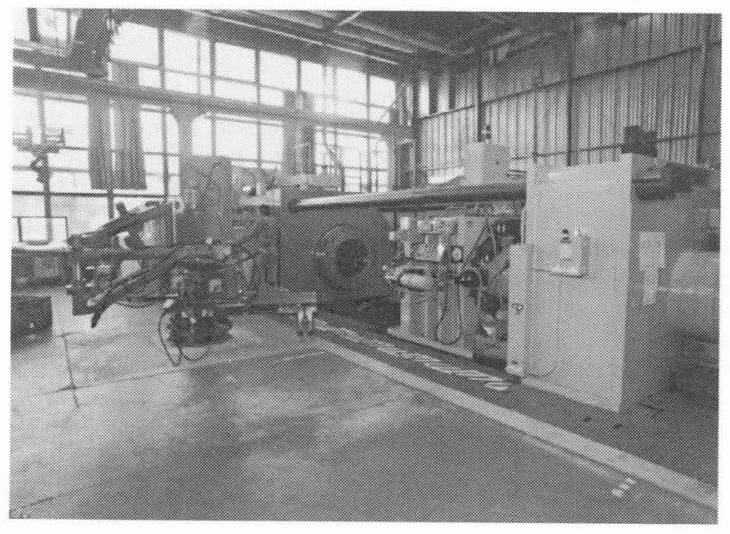

figure 15 : machine MTI - M480 utilisée par SNECMA pour rassemblage des rotors HP.

\section{Soudage : conditions et résultats}

Les paramètres de soudage à échelle 1 ont été définis, à partir des résultats obtenus à échelle réduite, par mises au point successives en recherchant une plage de consommation matière comprise entre 3 et $5 \mathrm{~mm}$, tout en cherchant à réduire au minimum la vitesse de soudage pour limiter lépaisseur de la zone blanche (figure 9) ; les épaisseurs à souder sont comprises entre 6 et $10 \mathrm{~mm}$ et les diamètres moyens entre 280 et $380 \mathrm{~mm}$. Les paramètres finalement retenus sont peu différents de ceux déterminés, pour des géométries similaires, par les essais à échelle réduite (\$ $\|$ i- 1 , figure 6$)$ et confirment la réduction de l'énergie nécessaire au soudage quand le diamètre moyen augmente. 
Les micrastructures des liaisons obtenues sont également similaires à celles correspondant aux essais à échelle réduite. Nous obtenons, pour répaisseur $6 \mathrm{~mm}$, une zone blanche d'environ $100 \mu \mathrm{m}$ abrs qu'elle est d'environ $400 \mu \mathrm{m}$ pour la liaison d'épaisseur $10 \mathrm{~mm}$, les capacités de la machine ne permettant pas, pour cette dernièré épaisseur, de réduire suffisamment la vitesse de soudage.

La mise au point a conduit à faire évoluer les outillages pour satisfaire aux contraintes géométriques et plus particulièrement à celle du défaut de perpendicularité provoqué par le retrait thermique de la zone de liaison lors de son refroidissement après soudage. Cette évolution d'outillage a mis en évidence, pour une géométrie de pièce constante, de fortes évolutions de la consommation-matière qui imposent de figer la géométrie de routillage dans la gamme de fabrication validée.

Les dispersions géométriques mesurées sur un ensemble de 6 rotors sont acceptables et de valeurs suivantes :

- C.M < $0.3 \mathrm{~mm}$ (elle n'est que de $0.1 \mathrm{~mm}$ pour des rotors en alliage de titane de dimensions similaires),

- défauts de coaxialité et de perpendicularité : $<0.25 \mathrm{~mm}$.

\section{Opérations post-soudage}

Sur les superalliages de nickel, il est impératif d'usiner les bourrelets, internes et externes, ot leurs défauts associés (entaille, cavitations) avant d'effectuer lo traitement thermique post-soudage; la réalisation de ce dernier sur état non réusiné a en effet conduit systématiquement à des fissurations traversantes, perpendiculaires au plan de joint et uniformement réparties sur toute la liaison. Ces fissures sont reliées aux défauts présents dans les bourrelets à rétat brut de soudage et aux contraintes résiduelles tangentielles (figure 14) qui sont généralement accentuées, sur les supérailiages de nickel, lors d'un revenu post soudage (fissuration au revenu [9]).

Le trailement thermique post soudage est donc réalisé après assemblage complet du rotor et réusinage de chacun des bourrelets sur une profondeur permettant d'éliminer les zones défectueuses.

Le moyen de contrôle non destructif le mieux adapté à ce procédé est le contrôle par ultrasons en ondes longitudinales orientées perpendiculairement au plan de joint. En effet, il est observé que les défauts potentiels, dûs à une consommationmatière insuffisante ou à une pollution accidentelle du matériau de base, sont systématiquement alignés le long du plan de joint.

\section{Maîtrise du procédé - assurance qualité}

La programme d'assurance qualité correspondant est de mise en ceuvre aisée comparativement à celui du soudage par faisceau d'électrons, du fait de la grande simplicité du procédé F.I. Une fois validée, par une dissection appropriée, la qualité d'un ensemble donné réalisé sur une machine donnée, avec un outillage figé et suivant un ensemble précis de conditions de soudage, ressentiel de Passurance qualité d'un ensemble soudé F.I est obtenu en vérifiant que la consommation-matière résultante est située à lintérieur de la fourchette validée. Cette approche peut être affinée en comparant la courbe d'évolution de la C.M en fonction du temps à un domaine d'évolution validé. Les contrôles associés à la qualité de la machine sont un étalonnage régulier de la pression et de la vitesse de rotation de la broche qui peuvent être completés par des essais spécifiques permettant de suivre une éventuelle dégradation mécanique de cette dernière. A titre d'exemple, il est possible, avant chaque campagne de soudage, d'assembler une éprouvette de référence, de forme simple et en matériau à qualité bien définie, qui aura été préalablement caractérisée au cours de la validation, dont révolution éventuelle de la consommationmatière au cours des campagnes successives sera révélatrice de l'évolution mécanique de linstallation de soudage.

\section{IV - MODEUSATION DU SOUDAGE F.I}

De façon plus générale, la mise sous contrôle du procédé passe par le développement d'un outil de modélisation. Cette démarche, prolongeant colle mise en ceuvre dans le domaine du forgeage, est çci rendue d'autant plus nécéssaire du fait des gradients de température et de déformation très sévères dans la zône soudée, de rextrème difficulté des mesures de température en cours d'assemblage et de la philosophie d'assurance qualité visant à garantir la conformité de la liaison par le contrôle des paramètres du procédé.

Deux axes d'études ont été définis : révolution de la structure métallurgique de lalliage au cours du cycle thermomécanique subi et le développement des outils numériques adaptés.

Des travaux déjà mentionnés [3] ont fourni les bases expérimentales et un cadre de modélisation de la mise en solution des différents précipités au cours de cyclages thermiques rapides. Par ailleurs le comportement rhéologique à chaud associé a été identifié. Enfin, des fonctionnalités nouvelles ont dû être introduites dans FORGE2 [2] afin de prendre en compte les effets d'inertie, de modéliser le frottement au plan de joint, et de developper les critères de remaillage local.

Une première version du logiciel a été implantée à SNECMA, destinée à la validation par comparaison avec les essais expérimentaux à échelle réduite.

L'objectif final est lutilisation de ce code par les services Méthodes en vue de diminuer les coûts et les délais de développement et d'industrialisation en permettant de diminuer le nombre d'éssais de mise au point de gamme et de maitriser les changements d'échelle; des gains de qualité sont également attendus de la quantification par le calcul des effets des variations de paramètres du procédé de même que de la maitrise par le modèle de la structure metallurgíque des produits. 


\section{V - CONCLUSIONS}

Les études de mise au point du soudage par friction inertielle des superalliages, ASTROLOY puis N18, nous ont permis de définir les relations existant entre la géométrie des sections à souder, la microstructure du matériau de base, les paramètres de soudage ef la microstructure résultant de la liaison et ses caractéristiques mécaniques, dans le domaine de fonctionnement d'un compresseur HP et pour différents traitements thermiques post-soudage.

II a été ainsi possible:

- de construire une gamme de fabrication permettant de satisfaire des critères géométriques sévères ainsi qu'un programme d'assurance qualité d'une grande simplicité,

- de définir les paramètres opératoires permettant de satisfaire aux critères mécaniques des rotors de compresseur HP actuelement soudés à SNECMA et de préciser la direction des évolutions à réaliser, sur les paramètres opératoires et/ou sur les traitements thermiques post-soudage, pour pouvoir satisfaire aux spécifications mécaniques plus sévères des moteurs futurs.

\section{REFERENCES}

[1] Brevet français $N^{\circ} 86016041986$

[2] MOAL A, "Modélisation numérique par la méthode des éléments finis du soudage par friction inertielle", thèse Ecole des Mines de Paris - 1992.

[3] SOUCAIL M., " Etude théologique et microstructurale de rASTROLOY en vue de la modélisation du soudage par friction inertielle", thèse Ecole des Mines de Paris - 1992.

[4] CLAD T., communication privée.

[5] MOSSER P.E., communication privée.

[6] NESSLER G. et al., " Friction welding of titanium alloys", Welding Journal, sept. 1971, WRS pp $379-385$.

[7] Rapport interne SNECMA, 1992.

[8] Rapport interne SNECMA, 1991.

[9] KOU S., Welding metallurgy", Eds John Wiley \& Sons, 1987, p. 306. 